\title{
Correspondence
}

\section{Arterial oxygen saturation in cigarette smokers following general anaesthesia}

\section{To the Editor:}

A.R. Tait et al. ${ }^{\prime}$ in the May, 1990, issue of the Journal conclude, based on their Table III, that the "severity of hypoxaemia was significantly greater in the smoking group than in the non-smoking group."

Table III, as presented, is an example of rank ordered data, with smoking status as the independent variable and $\mathrm{SaO}_{2}$ as the dependent variable. I have recalculated the table to replace percentage by number of subjects.

TABLE III

\begin{tabular}{lllcc}
\hline & \multicolumn{4}{c}{$\mathrm{SaO}_{2}$} \\
\cline { 2 - 5 } & $\leq 84$ & $85-89$ & $90-94$ & $\geq 95$ \\
\hline Smokers & 3 & 3 & 7 & 6 \\
Non-smokers & 0 & 4 & 10 & 10 \\
\hline
\end{tabular}

The most appropriate statistical test for this data is the Mann-Whitney U test, ${ }^{2}$ which can be done manually and is included in most statistical packages. This test compares each of the smokers with each of the non-smokers, a total of $19 \times 24=456$ comparisons. If there is no difference half the comparisons $\left(U_{E}=228\right.$ ) should favour each group. The actual test statistic $U$, which is easily calculated as 273 , can be interpreted in two ways. First the probability that a smoker, chosen at random, will have a lower $\mathrm{SaO}_{2}$ than a non-smoker, also chosen at random, is $273 / 456$ or 0.6 . Not much greater than chance, which would be 0.5 . Secondly one can calculate the standard deviation and assess the statistical significance of $U$. The exact two-tailed probability is $0.25 ;^{3}$ the approximate $P$, calculated by hand, is 0.28 . Although the Chi-square and Fisher's exact tests are not appropriate for this data, they also lead to non-significant P-values. It is therefore difficult to understand how the authors have reached their conclusions.

With regard to Table $I 1$, they conclude that the "magnitude of the decrease in $\mathrm{SaO}_{2}$ was significantly greater in the smoking group." Although mean $\mathrm{SaO}_{2}$ values in the PACU look like they should be significantly different, the baseline values from the $O R$ are also different.

Most of us believe that cigarette smoking increases the risk of anaesthesia. Unfortunately this paper does not provide us with scientifically valid estimates of that risk. Although the mean $\mathrm{SaO}_{2}$ may be lower during transport in smokers, the probability of any randomly selected smoker being at greater risk than a non-smoker is too close to chance to serve as a criterion for therapeutic decisionmaking. Other factors must be considered in order to individualize treatment. If, based on this study, you advocate $\mathrm{O}_{2}$ for smokers, then rationally you must also advocate $\mathrm{O}_{2}$ for non-smokers.

\section{W.A. Tweed MD FRCPC \\ National University of Singapore}

\section{REFERENCES}

I Tait AR, Kyff JV, Crider $B$ el al. Changes in arterial oxygen saturation in cigarette smokers following general anaesthesia. Can J Anaesth 1990; 37: 423-8.

2 Moses LE, Emerson JD, Hasseini $H$. Analyzing data from ordered categories. N Engl J Med 1984; 311: 442-8.

3 Statgraphics, Statistical Graphics Corporation, 2115 East Jefferson St., Rockville, MD, USA 20852.

\section{Spinal narcotics}

To the Editor:

I am concerned about the current popularity of neuroaxial opioids and opiates in obstetrics as revealed in the editorial by Dr. Writer.' In my opinion, the danger of reactivation of previous serious CNS or ophthalmic herpes infection remains an unassessed, but potentially absolute, contraindication to the use of epidurally or intrathecally administered opioids or opiates. Given the rarity of serious extragenital infections none of the sample sizes of the studies done to date reassure me that reactivation of ophthalmic herpetic infection and its possible corneal destruction might not occur. Herpes keratitis is not unknown in the obstetric population and one can only speculate as to the risk in the immunocompromised obstetric patient.

Whether one is a "declining dowager" or a fecund female it appears to me to be a reasonable precaution to 
include inquiries about previous serious extragenital herpes infection and to avoid the use of epidural or intrathecal opioids and opiates in those rare patients who have a positive response.

\section{Beriault MD \\ Grace Hospital \\ Calgary, Alta.}

\section{REFERENCE}

1 Writer WDR. Epidural morphine for post-Caesarean analgesia. Can J Anaesth 1990; 37: 608-11.

\section{Nasotracheal intubation using Bullard ${ }^{(\mathbb{T}}$ laryngoscope}

To the Editor:

Several methods are available for difficult endotracheal intubation but much time and skill are required. The method using the Bullard laryngoscope (Figure), which is an anatomically shaped rigid fibreoptic instrument to aid indirect laryngoscopy, is one of the solutions. Saunders and Geisecke ${ }^{1}$ presented a clinical assessment of the adult Bullard laryngoscope. They used intubating forceps to grasp the tube through the Murphy eye and introduced the tube through the glottis. If the forceps are not used, as Bjoraker ${ }^{2}$ suggested, a styletted endotracheal tube formed to mimic the shape of the blade can be used for oral approach. However, the oral approach with intubating forceps or stylet requires considerable practice before the technique is reliable because the space for manipulating the endotracheal tube is narrow. Consequently, the smooth insertion of the blade and the endotracheal tube together through the pharynx is not easy. To improve this we propose a new method of intubation using the Bullard laryngoscope, which requires no special skill.

Our method is as follows: the Bullard ${ }^{50}$ laryngoscope is inserted orally to visualize the glottis, following which an endotracheal tube is inserted through the nose and advanced to the glottis. The positional relation of the glottis and the tube is observed through the laryngoscope throughout intubation, and the direction of the tube tip is changed if necessary. We compared three kinds of endotracheal tubes, i.e., tubes with strong curvature and moderate hardness, styletted tubes, and directional tip tubes (Endotrol ${ }^{\text {(it) }}$ (Figure). All were useful but the directional tip tube (Endotrol ${ }^{60}$ ) was the best for quick and non-traumatic intubation.

The advantages of our method lie in the good view of

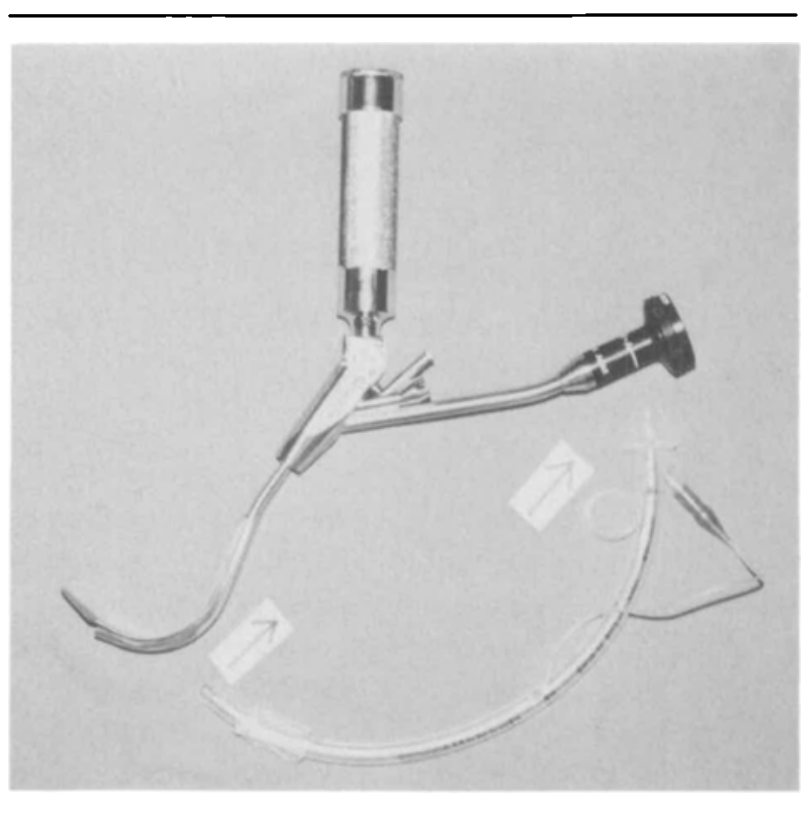

FIGURE The adult Bullard laryngoscope is shown with a standard two-C-cell laryngoscope handle attached and its viewing arm with eyepiece extended to the right. The directional tip tube (Endotrol(t) is shown below the laryngoscope. The tube tip bends to the direction of the left arrow when the wire ring is pulled to the direction of the right arrow.

the glottis which is obtained with the Bullard laryngoscope and the anatomically easy access to the glottis by nasal insertion of an endotracheal tube. The direction of the tube tip can be changed without disturbing the view of the larynx. In addition, the laryngoscope can be held in the left hand and only $6 \mathrm{~mm}$ of oral opening is required to place the blade.

We found that tracheal intubation can be achieved easily by this method of nasal intubation using the Bullard laryngoscope in difficult airway patients, and hope that others will try the method.

Toshiyuki Shigematsu MD

Noriko Miyazawa MD

Tomoko Yorozu MD

Department of Anesthesiology,

Tokyo Metropolitan Ohtsuka Hospital,

2-8-1 Minami Ohtsuka,

Toshima-ku,

Tokyo, 170, Japan

\section{REFERENCES}

1 Saunders PR, Geisecke AH. Clinical assessment of the adult Bullard laryngoscope. Can J Anaesth 1989; 36: S118-S119.

2 Bjoraker DG. The Bullard intubating laryngoscopes. Anesthesiology Review 1990; 17: 64-70. 